\title{
Interaction Weaknesses of Personal Navigation Devices
}

\author{
Markus Hipp*, Florian Schaub*, Frank Kargl ${ }^{\dagger}$, Michael Weber* \\ *Institute of Media Informatics \\ Ulm University \\ 89069 Ulm, Germany \\ \{firstname.lastname\}@uni- \\ ulm.de \\ †DIES Research Group \\ University of Twente \\ P.O. Box 217 \\ 7500 AE Enschede, NL \\ f.kargl@utwente.nl
}

\begin{abstract}
Automotive navigation systems, especially portable navigation devices (PNDs), are gaining popularity worldwide. Drivers increasingly rely on these devices to guide them to their destination. Some follow them almost blindly, with devastating consequences if the routing goes wrong. Wrong messages as well as superfluous and unnecessary messages can potentially reduce the credibility of those devices. We performed a comparative study with current PNDs from different vendors and market segments, in order to assess the extent of this problem and how it is related to the interaction between device and driver. In this paper, we report the corresponding results and identify multiple interaction weaknesses that are prevalent throughout all tested device classes.
\end{abstract}

\section{Categories and Subject Descriptors}

H.1.2 [User/Machine Systems]: Human factors; H.5.2 [User Interfaces]: Evaluation/Methodology; H.5.2 [User Interfaces]: Ergonomics

\section{General Terms}

Human Factors, Experimentation

\section{Keywords}

HCI, personal navigation devices (PND), study

\section{INTRODUCTION}

Automotive navigation systems have become an assistant technology many drivers rely on. Navigation systems are either directly integrated in the vehicle or come as add-on solutions, like dedicated portable navigation devices (PNDs), or smartphone software solutions. By navigation system we refer to a device or component that performs routing decisions locally, in contrast to online routing services where some or all routing calculations are performed by servers online. In this work, we focus on PNDs, due to their widespread adoption and global market share [5].

Copyright held by authors.

AutomotiveUI'10, November 11-12, 2010, Pittsburgh, Pennsylvania. ACM 978-1-4503-0437-5
Frequent drivers, business travelers, vocational drivers, and families entrust their navigation systems with finding a suitable route to their destinations. Nowadays, some drivers follow them almost blindly, i.e., by reacting instantaneously to driving directions announced by the device. Such blind trust can lead to dangerous situations as underlined by anecdotal reports of people driving their cars into a river because they followed the commands of their navigation system too literally [7]. However, the case of a German driver who caused an accident by turning around on a highway because his satellite navigation systems told him so [6] underlines the seriousness of the problem. Hanowski et al. [4] were able to show that the confidence placed in the commands of a navigation system and its credibility increases with the driver's unfamiliarity with the environment. They also showed that the driver's self-confidence decreases in such situations. Thus, the further away drivers are from their known environment, the more they rely on their navigation systems and trust their commands. In such situations drivers may also become susceptible to gullibility errors [3], which could result in them acting upon erroneous commands that they would most likely reject under normal conditions.

\subsection{The Credibility Issue}

Apparently, credibility is an important aspect of the problem at hand. Fogg and Tseng [3] distinguish between different types of credibility of software systems. Presumed credibility is based on general assumptions about the functionality of a system, surface credibility is attributed based on the impression made by the hardware and interface design, reputed credibility stems from the recommendations and opinions of third parties, while experienced credibility is based on earlier personal experiences. The first three aspects are prevalent in forming the initial credibility a driver attributes to the navigation system. However, experienced credibility evolves over time. While the first three credibility aspects in combination with unfamiliar environments may facilitate gullibility errors, the consequence of such an error will almost certainly destroy experienced credibility of the navigation system. Mistrust towards future commands of the device will be the result.

In familiar environments, a slightly different situation unfolds. Drivers feel more confident and due to their background knowledge can better judge the correctness of navigation commands, at least to a certain extent. Therefore, the danger of drivers acting upon erroneous commands should decrease. But in a familiar environment drivers also 
form their own (not necessarily correct) belief what the best route would be in a given situation. A mismatch between the planned route of the navigation system and the driver's belief of the best route is possible. Drivers may deliberately deviate from the suggested route to evade traffic jams or to make a purposeful detour, e.g., to stop at the grocery store, or because they think they know a shortcut. Typically, navigation systems react by issuing commands to lead the driver back onto the planned route or a newly calculated alternative route. But because the driver deliberately deviated from the route such messages will be considered annoying especially if they occur repeatedly. It is likely that such annoying or superfluous commands also have a negative effect on the experienced credibility of the device.

Another feature that may cause similar effects is the integration of dynamic traffic information in the routing process. Some navigation systems can receive dynamic traffic messages from different sources to inform the driver about traffic obstructions ahead and to suggest a detour. While potentially a useful service, the presentation of such information can impact the experienced credibility of the system. For example, displaying the age of a traffic message would be a useful indicator to judge its relevance and accuracy, while its absence may foster gullibility errors, if the navigation system suggests a detour to avoid a traffic jam based on information that is hours old. Furthermore, the benefit of a detour needs to be apparent to drivers and should match their understanding of an appropriate alternative route.

Thus, the credibility of navigation systems can be reduced by gullibility errors, but superfluous or annoying messages can also impact it negatively. The experienced credibility can potentially degrade to such a level that incredulity errors occur [3]. Drivers would ignore navigation commands because they doubt their accuracy, even if following these commands would actually benefit them. This of course can lead to a further decrease of experienced credibility. In addition, the exchange of negative experiences between customers can also decrease the reputed credibility of a product. Therefore it is in the best interest of vendors to ensure that not only presumed and surface credibility remain high but also experienced and reputed credibility.

\subsection{Contribution \& Outline}

In this work, we analyze the extent of interaction issues of PNDs, which may cause credibility deterioration. In particular, we focus on the frequency of superfluous and erroneous messages and the integration of dynamic traffic warnings. We performed a comparative study with PNDs of different vendors under real world conditions to assess their interaction behavior with the driver. Based on the results, we identified weaknesses in the interaction process and particularly problematic scenarios. As part of a structured research approach, these results will be used in future work to assess credibility deterioration in navigation systems and to develop optimized interaction processes for such devices.

Section 2 provides background information on navigation systems with a focus on information sources that impact routing decisions and navigation commands. Section 3 outlines the setup of our study, and Section 4 introduces the applied metrics. Results are presented in Section 5. A dis- cussion of these results and the identified weaknesses follows in Section 6. Section 7 concludes the paper.

\section{BACKGROUND}

Navigation systems draw information form multiple sources to make routing decisions. Besides the current position, which is usually determined with GPS, static map data stored in the device constitutes a main information source. Streets are represented by directed graphs, where nodes represent intersections or fixed points on the map and edges represent streets connecting nodes. Directed multigraphs are used to represent multiple lanes, i.e., two nodes can be connected with multiple edges. Direction of a lane is represented by edge direction. Additional attributes can express the type of streets, speed limits, etc. Edges are weighted. In the easiest implementation, a weight represents the length of a street segment, but weights may also be time-dependent to indicate different traffic situations over the day. The latter enables devices to optimize routes for the current time of day. To identify the optimal route, the cheapest path from start to finish has to be found. Different graph search algorithms exist to calculate these paths, for example, Djikstra's $\mathrm{SPF}[2]$ or $\mathrm{A}^{*}[1]$.

Another information source for PNDs are dynamic traffic messages. Service providers as well as some PND vendors, like TomTom ${ }^{1}$ or Garmin ${ }^{2}$, collect driving information from their users to generate time-dependent traffic information. TomTom devices with $i Q$-Routes come preloaded with a database filled with the information from over 10 billion driven kilometers. This additional information is used to calculate the most effective route for the driver depending on the current time of day, which might not necessarily be the shortest. Garmin offers nüLink! online services, which provides traffic information and Internet search functionality to models equipped with an integrated GSM module.

In addition to proprietary solutions, different services exist to broadcast information about traffic jams, accidents, or other incidents to vehicles. The most common service is the Traffic Message Channel $(T M C)$, which is a particular service of the Radio Data System (RDS), now under control of the Traveller Information Services Association ${ }^{3}$ (TISA). TMC provides travel information via signals broadcast over the FM-Data-Channel. TMCpro is an improved derivative of the $T M C$ service, operated as a commercial service by Navteq. ${ }^{4}$ While $T M C$ relies mainly on reports by drivers, TMCpro utilizes real-time traffic data automatically generated by data sensors, which are installed on highways. The process of extracting traffic warning from the gathered data is automated and not subject to editorial control. As the extension possibilities of TMC and TMCpro are limited, the TPEG $G^{5}$ standard, also maintained by TISA, is able to provide more precise information about traffic jams or accidents. TPEG will replace $T M C$ as the standard service to transmit dynamic traffic information.

\footnotetext{
${ }^{1}$ TomTom website: http://www.tomtom.com/

${ }^{2}$ Garmin website: http://www.garmin.com/

${ }^{3}$ TISA website: http://www.tisa.org/

${ }^{4}$ Navteq website: http://www.navteq.com/

${ }^{5}$ Transport Protocol Experts Group
} 
Navigation system manufacturers also provide other features to improve user interaction. For example, in order to provide more precise announcements, some devices use textto-speech technology to convert natural language text into speech. This enables the inclusion of street names and other dynamic attributes into announcements, which helps the driver to relate the provided information to the real world environment.

\section{STUDY DESIGN}

The goal of our study is the comparison of the interaction behavior of different navigation systems under real world driving conditions. We focus on the frequency of navigation commands and messages, as well as the quality of the provided messages. See Section 4 for the employed metrics. But first, we describe the analyzed devices and the driving scenarios of the study.

\subsection{Devices}

For the study, only portable navigation devices (PNDs) have been considered as they constitute the most commonly used type of navigation systems [5]. Due to their portability they also facilitate comparison under the exact same conditions, as multiple PNDs can be installed in one car.

We want to gain a reasonable overview of the interaction concepts currently prevalent in the market and, therefore, selected devices covering bottom-range $(\$)$, mid-range $(\$ \$)$, and top-range $(\$ \$ \$)$ segments of the German PND market. The analyzed devices are listed in Table 3.1. In the remainder of this paper, we will refer to the devices by their designated labels.

Three of the five test devices support the reception of dynamic traffic information via TMC or TMCpro. Tomtom further supports the iQ routes system, while Garmin only supports the Garmin operated nüLink! service. Pearl, as a bottom-range device, is the only navigation system that does not come directly with a receiver for dynamic traffic information services. Note that text-to-speech is only supported by the mid-range and top-range devices.

\subsection{Driving Scenarios}

The performed test drives were split into predefined driving scenarios to analyze the behavior of the navigation systems in different situations. During the test drives, the devices were mounted on a common board inside the vehicle. A setup of two cameras monitored the audiovisual output of the navigation systems as well as the current road situation. Additionally, two persons transcribed navigation commands and audiovisual messages issued by the devices. Fig. 1 depicts the in-car setup. In preparation for each scenario, all tested devices were set to the same destination, and it was verified that the devices calculated a similar route $\left(R_{c}\right)$. Depending on the driving scenario (see below) deliberate deviations from the route were planned and performed, resulting in a different driven route $\left(R_{d}\right)$. The driven route $R_{d}$ is supposed to reflect the intentions of a driver and the driver's belief of the correct route.

The driving scenarios have been designed to specifically analyze how the devices react in common driving situations.

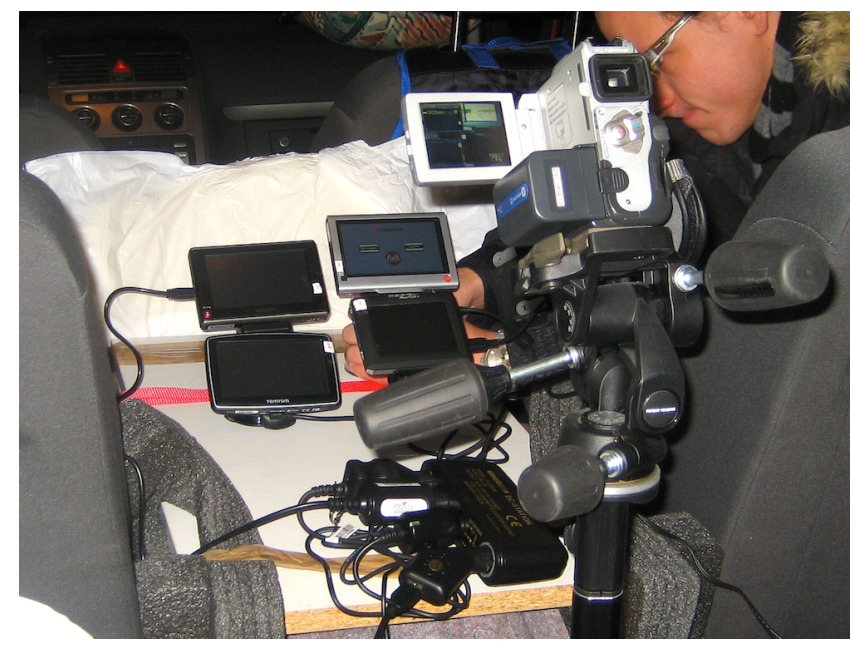

Figure 1: In-car setup of the navigation systems.

Thereby, a balanced mixture of urban, suburban, rural, and highway road usage was factored in.

Detour (city). The calculated route $R_{c}$ is left for a deliberate urban detour. A typical reason for such driving behavior would be the need to run quick errands before embarking onto the actual journey, e.g. to stop by a grocery store or drop children off at school. In this scenario, the driven $R_{d}$ and the calculated route $R_{c}$ do not match. The main purpose is to analyze how devices react to deviations from the route. Road usage in this scenario is mainly urban and suburban.

Highway. On the highway, commands of the navigation systems are followed to analyze normal operation. In addition, a stop at a petrol station is simulated to see how navigation systems react.

Inner city. In this scenario, the routing behavior of the devices close to a destination is analyzed. Devices should support the driver in finding the destination, yet provide freedom to search for a parking spot in proximity of the destination. Urban roads dominate this scenario.

Detour (rural). Similar to the detour (city) scenario, the driver deviates from the route on purpose. However, the detour is chosen over rural roads in such a way that there is no obvious alternative route the PNDs could switch over to. A typical scenario for this case would be the spontaneous decision to visit some touristic attraction or friends who live close to the original route.

Dynamic traffic warning. Here, we deliberately travel a chosen route during rush hour with many traffic jams along the route. The aim of this scenario is the analysis of the integration of dynamic traffic warnings. Of special interest is the presentation of the warning message, associated information, and potential alternatives to the driver.

The first four scenarios were combined in one route from Ulm University, just outside the town of Ulm, to a location in the inner city of Munich, and back. Fig. 2 gives an 
Table 1: Analyzed Devices

\begin{tabular}{llccc}
\hline Label & Manufacturer and model & Text-to-speech & Dynamic traffic services & Price segment \\
\hline Becker & Becker Traffic Assist Z205 & yes & TMC, TMCpro & $\$ \$ \$$ \\
Garmin & Garmin nüvi 1690 & yes & nüLink! & $\$ \$ \$$ \\
Falk & Falk F6 & yes & TMC, TMCpro & $\$ \$$ \\
Tomtom & TomTom XL Europe & yes & TMC, iQ Routes & $\$ \$$ \\
Pearl & Pearl V35-1 & no & - & $\$$ \\
\hline
\end{tabular}

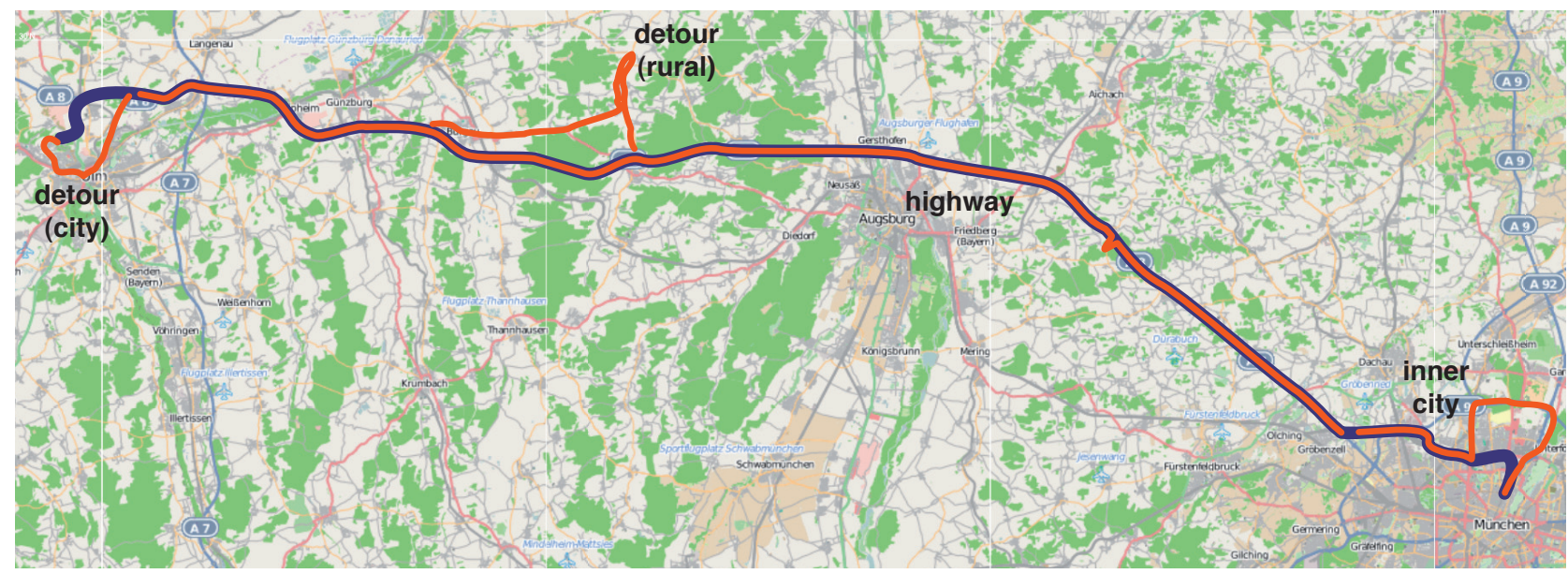

Figure 2: The calculated route $R_{c}$ (blue) and the different scenarios (red). Map data: OSM.org

overview of the planned route $R_{c}$ (blue), the different scenarios, and the driven route $R_{d}(r e d)$. The dynamic traffic warning scenario was performed and tested separately, as one had to dynamically look for suitable traffic jams.

\section{METRICS}

To allow an objective comparison of the different devices under test, we define specific metrics for measuring their behavior. First of all, we distinguish between acoustic and visual messages and navigation commands. Visual messages contain all information that is shown on the display. Acoustic messages are voice instructions from the system, which either support visual messages or provide distinct information. A navigation command may consist of an acoustic or visual message, or both. For example, a typical navigation command for turning right in 300 meters would consist of the acoustic message "turn right in 300 meters" and a visual message on the display composed of an arrow pointing to the right and the distance below or next to it. Animations that convey device activity are also counted as visual messages, e.g. two animated turning arrows when the route is recalculated.

In the analysis, we distinguish observed navigation commands according to message correctness and driver anticipation. We define message correctness to reflect the correctness of the issued navigation command in terms of the driver's intention. This means a message is correct, if its content conforms with and supports the driver's belief of the correct route. All other messages are labeled false. In our study, all events that correspond to the route intended by the driver $R_{d}$ are labeled as correct.

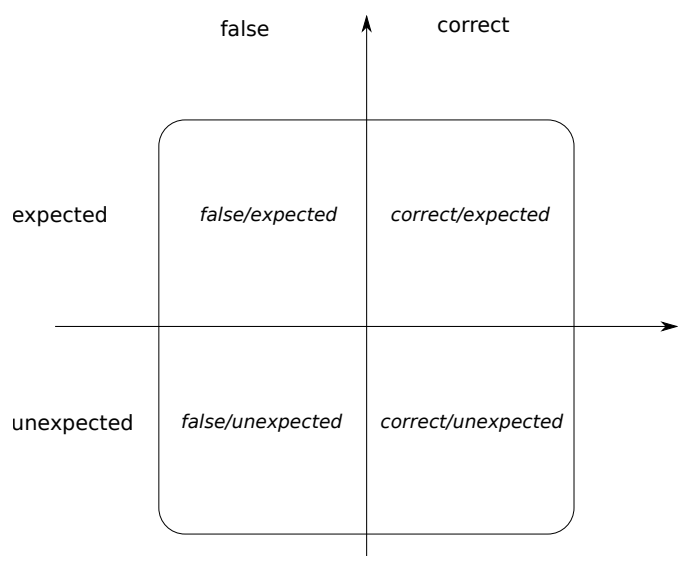

Figure 3: Definition of four message categories.

Driver anticipation measures if the driver can expect the message or if the message comes as a surprise, i.e., is unexpected. Expected messages are predictable in the sense that they fit the current situation and align with the behavior of the driver, but not necessarily with the driver's intention. They constitute the majority of all messages. All other messages appear unexpected. In our study, a message is labeled expected if it supports the calculated route $R_{c}$ in the current situation.

Fig. 3 depicts the two axes and the four possible message categories. Correct/expected messages constitute desired navigation commands. Correct/unexpected messages provide correct information but are not anticipated in the current situation, e.g., the message "follow the road for 60 kilome- 


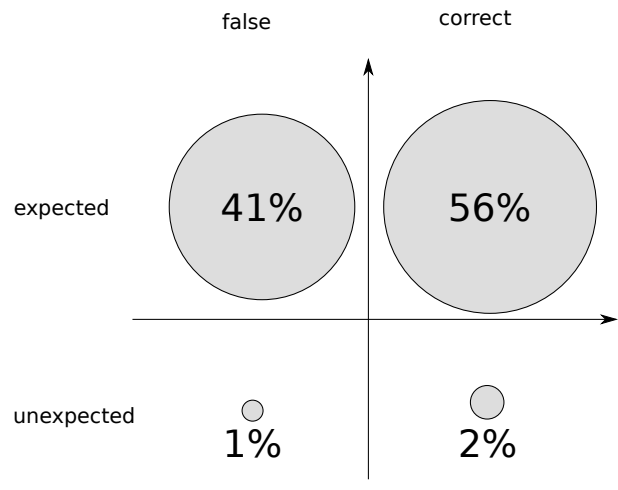

Figure 4: Average distribution of all observed messages.

ters" is correct but unexpected if the driver has been following this road for several kilometers already. False/expected messages occur when the driver deviates from the route. The message content does not conform to the driver's intention, but due to explicitly leaving the route such messages can be anticipated by the driver, e.g., repeating "turn around" messages. The instruction to turn around while driving on a highway falls in the category of false/unexpected messages.

\section{RESULTS}

In the following, we present the results of the performed study and analyze the behavior of the tested devices for each driving scenario.

In total, 162 kilometers where driven in 2:06 hours. In this time, 189 acoustic messages and 191 visual messages have been observed. Due to GPS radio reception problems, the Tomtom device had to be replaced to the side window, where it could not be captured by the camera anymore. However, the behavior of the Tomtom device was still observed and recorded manually.

The cumulative observation results over all devices and the first four driving scenarios are given in Fig. 4. Unexpected messages account only for $3 \%$ of all messages. The reasons for their occurrence are GPS radio reception problems. Thus, these messages are indeed dangerous but relatively rare. False/expected messages account for $41 \%$ of all observed messages, while merely $56 \%$ of all messages were correct/expected. While the extreme nature of the chosen driving scenarios degraded these values compared to a normal drive, it shows that PNDs react inflexible to changing driver intentions. Interestingly, all devices behaved quite similar irrespective of their price segment.

A detailed analysis of each driving scenario is provided in the following, highlighting typical reactions and behavior. The cumulative results for each scenario are summarized in Fig. 5.

\subsection{Detour (city)}

The $16 \mathrm{~km}$ long scenario was completed in 24 minutes. As expected, all devices called on the driver to turn around, as soon as the driver deviated from the planned route. The Falk called on the driver to turn around seven times (Becker five times, Pearl three times). As acoustic messages, Pearl and Becker used phrases like "turn left, then turn left". Only Falk used the words "please turn around".

With 41 acoustic messages, the Pearl was most active in this scenario, while the Becker acted very passive, with only six acoustic messages. Falk and Pearl produced nine false messages each, Becker only produced seven. Messages were false in the sense that they did not correspond to the driver's intention. Falk and Pearl recalculated their route nine times (indicated by visual messages). In case of the Pearl two of the recalculations resulted from GPS radio reception problems. The Becker performed six recalculations.

Fig. 5(a) gives the cumulative results for this scenario. The high percentage of correct/expected messages (57\%) results from the fact that all devices stopped issuing turn around messages and reverted to an alternative route $R_{c}^{\prime}$ as soon as that route became shorter than he orignal route $R_{c}$. Subsequently, mainly correct/expected messages were issued, because $R_{c}^{\prime}$ corresponded to $R_{d}$. Thus, the $40 \%$ false/expected messages occurred almost completely in the first minutes of this scenario.

\subsection{Highway}

In this scenario, we analysed the devices' behaviour during a highway trip, where the driver followed the calculated route, i.e., the driven route $R_{d}$ matched the calculated route $R_{c}$. As expected, all navigation systems remained passive and calm during the whole trip of 123 kilometers (76 minutes). The average number of visual messages was 10.6 , from which $74.4 \%$ have been correct (see Fig. 5(b)). There have been an average of 4.3 acoustic messages during the scenario. At one point, shortly before the end of the scenario, the calculated route $R_{c}$ of two PNDs differed from the others and the intended route $R_{d}$.

To simulate the refueling at a petrol station, the highway was left once. Due to the fact that the gas station was located near the shoulder of the highway, none of the devices corrected the displayed position but continued showing the car on the highway. When the trip was resumed, one device temporarily displayed an erroneous position and orientation for the car. Because the device believed the vehicle to be driving in the opposite direction, the driver was instructed to leave the highway at the next exit to turn around (false/unexpected message).

\subsection{Inner city}

During the inner city scenario (17 km, 19 minutes), reliable routing by all devices was observed. $92.5 \%$ of visual messages and all of the acoustic messages were correct (see Fig. 5(c)). The precise acoustic messages provided by the Falk device near intersections are notable. Announcements included the indication of direction and street names. For example, "In 500 meters, leave the highway towards B13 Munich, therefore keep right" in contrast to instructions like "After 800 meters, keep right, then keep left" issued by other devices. After reaching the destination, which all devices announced acoustically, all devices suspended routing and switched into free drive mode to support the driver in the search for parking. 


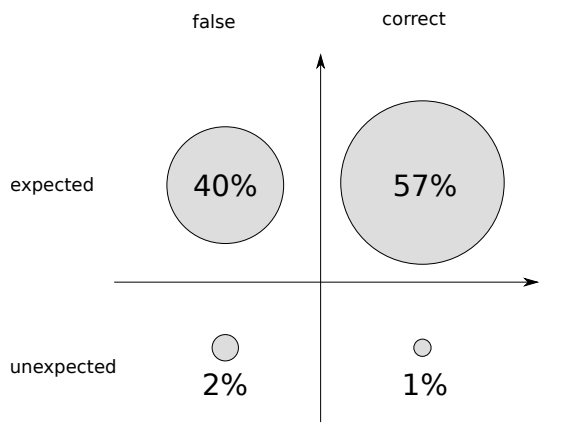

(a) Detour (city)

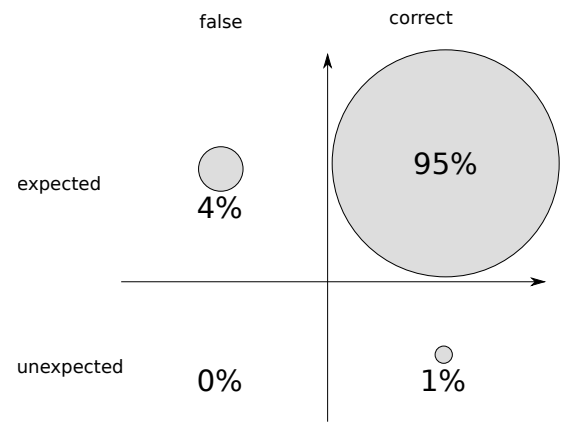

(c) Inner city

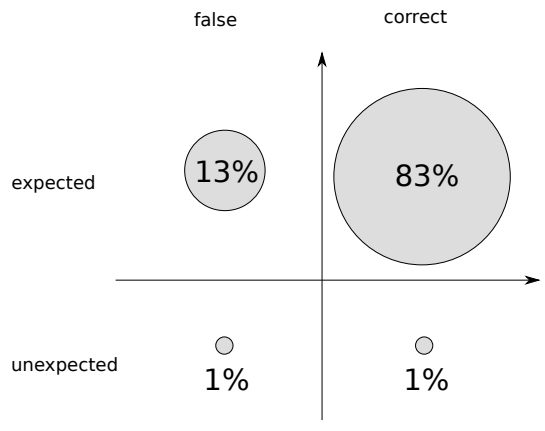

(b) Highway

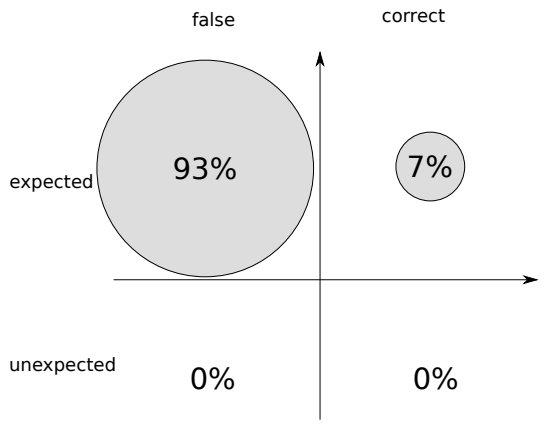

(d) Detour (rural)

Figure 5: Message Distribution for the different driving scenarios.

\subsection{Detour (rural)}

In this scenario, the driver deviated from the calculated route $R_{c}$ onto rural roads. Roads with few intersections were chosen to reduce potential alternative routes the devices could change to. The detour extended over $6 \mathrm{~km}(7$ minutes) before the driver turned around eventually. In this time, 6.3 route recalculations were performed on average. This corresponds to 1.16 recalculations per kilometer by Becker and Pearl, and 0.83 recalculations by Falk. None of the devices explicitly informed the driver about the reason of recalculation. Instead, turn around commands were repeatedly issued.

In combination with the large number of messages (one acoustic message every 37 seconds, one visual message every 57 seconds, on average) the behavior suggests, that the driver is rather stressed than supported by the devices.

\subsection{Dynamic traffic warning}

The integration of dynamic traffic warnings by the PNDs has been analyzed independently of the other four scenarios. We analyzed the behavior of devices in situations where warnings about traffic obstructions on the planned route $R_{c}$ appeared. Hereby, the focus was placed on the information the devices provide about the obstruction and how it is conveyed, as well as the decision support provided to the driver.

Due to different information sources for the dynamic traf- fic warnings, the listing of traffic information varied between devices, e.g., between Becker (TMC) and Garmin (nüLink!). Additionally, TMC messages can also differ strongly between devices, because, in Germany, radio broadcasting corporations are responsible for creating and updating traffic information. Each broadcasting corporation relies on it's own mix of different information sources, e.g., listeners, automobile associations like the German $\mathrm{ADAC}^{6}$, or police notifications.

If traffic warnings existed, the driver could call up a list of received warnings and their information on all devices. A detailed view for each message provides the reason for the obstruction (traffic jam, accident, etc.) and displays the approximate position of the obstruction. None of the tested devices provided information about a message's time of origin. When a dynamic traffic warning for an obstruction on the planned route $R_{c}$ is received, the tested devices call on the the driver to decide if the traffic jam should be circumvented.

As an example, Fig. 6 show the dialog of the Becker device. A traffic jam is depicted as a purple area on the planned route (red). A symbol on the right depicts the type of obstruction. The navigation system provides only one alternative to avoid the obstruction. Only approximations of the

\footnotetext{
${ }^{6}$ ADAC - Allgemeiner Deutscher Automobil-Club e. V.:
} http://www.adac.de 


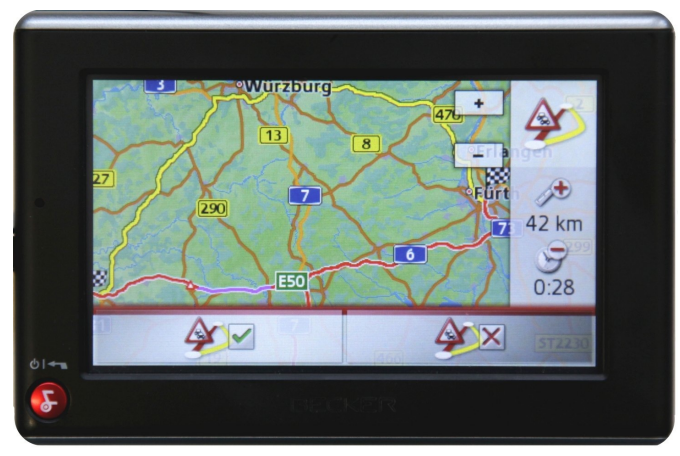

Figure 6: Recommended route to circumvent the traffic jam.

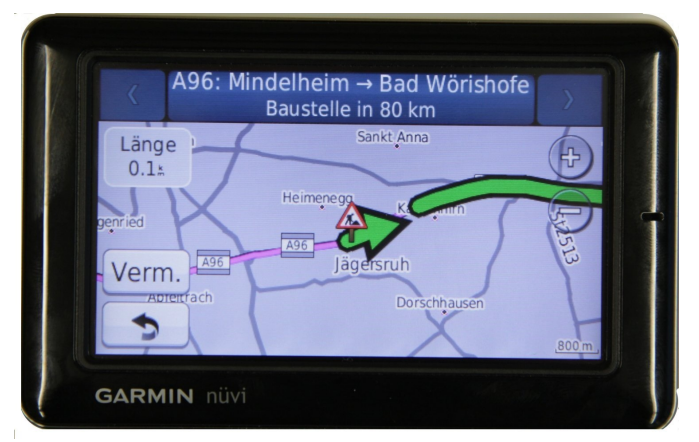

Figure 7: Display of an obstruction of traffic and the possibility to avoid this route.

saved time and the distance difference of the detour are provided as additional information. How the value of, in this case, 28 minutes has been determined is not obvious to the driver and not explained by the system. If additional information is available for the calculation of the saved time, e.g., characteristics of the traffic jam, it is not displayed to the driver. In the example, it is also not obvious and not explained, why this exact route (yellow) is recommended to avoid the traffic jam, while several other (presumably shorter) routes are discernible on the map (see Fig. 6).

However, other devices, e.g., the Garmin, do not even display the alternative route to the driver. Fig. 7 shows that the only information available to support the driver's decision is the length of the traffic jam $(0.1 \mathrm{~km})$ and the cause of the obstruction (construction site). Thereby, the degree of obstructions is displayed in different colors, e.g., green indicates a slight obstruction and red indicates a heavy obstruction.

In general, the tested devices provide not enough information about the obstruction and possible alternatives to enable the driver to make an informed decision about staying on the route or avoiding the obstruction. Unfortunately, the tested devices did also not provide a recommendation for the best choice.

\section{DISCUSSION}

In this Section, the results from the study are discussed and interaction weaknesses are summarized.
Currently, a driver can only influence the route prior to a trip by choosing between different route characteristics, e.g., shortest, fastest, or most economic route. Furthermore, the driver can choose to avoid toll roads or highways. Based on these adjustments, the navigation system calculates the route. At this point no reasons are given why this particular route is chosen, and the driver has no opportunity to compare this route to other alternatives. A comparison maybe performed internally by the system, but it is invisible to the driver. Almost all devices instantly started the route guiding. Only the Pearl displayed a general overview of the planned route before commencing navigation. While instantly switching to route guiding might save time, displaying a route overview can prevent mistakes due to wrongly selected destinations.

The study showed that PNDs are reliable and perform well in normal situations, when the calculated route $R_{c}$ matches the intended route $R_{d}$. However, while all PNDs issued correct and expected messages for navigation commands, several devices produced cryptic messages. Here, the top range devices performed notably better.

When the driver deviates from the calculated route $R_{c}$ on purpose, the devices are not able to dynamically adapt to the driver's decision. The driver is not properly informed about the deviation detected by the device, route recalculations occur mostly silently and are only accompanied by short visual messages (e.g. turning progress arrows). The navigation systems instruct the driver to turn around but give no indication why, i.e., to return to the calculated route $R_{c}$. Interestingly, only one device actually used the vocal instruction "turn around", while the others resorted to sequences of turn commands ("turn left, then left"). The latter behavior not only increases the number of messages but also does not convey the intention of the device to the driver. Furthermore, the driver has no possibility to inform the navigation system about its intention (deviation from the route) without performing multiple clicks in touch menus to abort the routing process.

The study showed further that in both scenarios where the driver left the route intentionally the navigation systems produced $56 \%$ false acoustic and $65 \%$ false visual messages in total, respectively. While those messages can be expected by the driver, because they intentionally left the route, the observed frequency and persistence of such messages suggests increased stress for the driver in already stressful situations. In contrast, the numbers of correct messages were considerable higher in scenarios, where the driver followed the calculated route $(93 \%$ correct acoustic and $88 \%$ correct visual messages).

Another critical point is the interaction concerning dynamic traffic warnings. While tested devices that support the reception of dynamic traffic warnings notified the driver about obstructions on the planned route, the provided information is often not sufficient for the driver to make an informed decision if the obstruction should be avoided or not. The driver's options for interaction are also very limited. The driver can either accept the calculated detour (which is not displayed by all devices) or continue on the current route. It remains to be analyzed what information needs to be displayed to 
actually support the driver in this decision and to improve system credibility in such scenarios.

In summary, major interaction weaknesses could be identified for the route selection, the purposeful deviation from routes, and the integration of dynamic traffic warnings. Although the study included devices of different price ranges, the issues were prevalent throughout all devices with minor variations.

We conclude that in order to improve credibility, the interaction between the driver and the system must be improved. The driver should be able to comprehend system decisions, e.g., why a specific alternative route has been selected and not another. At the same time, devices should enable the driver to interact with them in order to convey changes in driving intention. With improved bidirectional information interchange, the amount of false messages could be potentially reduced and the devices could better adapt to drivers.

\section{CONCLUSION}

In this work, we studied the interaction behavior of several current personal navigation devices. We designed driving scenarios to analyze the behavior of PNDs from different categories in specific situations. All devices have been tested in parallel to ensure the same conditions.

The results of the study support our hypothesis that PNDs perform well as long as drivers follow their directions, but fail in deviating situations. As soon as the driver leaves the calculated route, all devices show interaction weaknesses. They issue cryptic commands ("turn left, then left") repeatedly, without communicating the actual problem to the driver, e.g., that the driver left the calculated route. While this behavior is desired to some extent when the driver leaves the route by accident, it becomes an annoyance in situations where the driver acts on purpose. Current PNDs are not flexible enough to cater for both situations. We argue that the root cause of these problems is the lack of interactivity with the driver en route. While vendors constantly work to improve routing functionality, as well as output mechanisms (e.g., text to speech), what is missing in today's devices are information exchange loops between driver and device. The navigation system does not expose enough information about its internal decisions. Instead, they should offer meaningful information about commands and route alternatives to empower drivers to understand why a certain route has been recommended. Otherwise navigation systems risk the loss of credibility with the driver.

At the same time, drivers have neither explicit nor implicit means to communicate their dynamic intentions to the navigation device.
They can only influence routing beforehand by specifying a vague route preference, e.g. fastest route. We claim that such static settings are not sufficient to capture a person's driving habits. Instead, navigation systems should utilize available sensors to implicitly infer the driving behavior over time and adapt accordingly. Furthermore, by offering meaningful interaction opportunities and alternatives, navigation systems could engage in a dialog with the driver, better adapt to the driver, and maintain high credibility. However, great care is required in interaction design to ensure that the driver can concentrate on the primary task - operating the vehicle.

We are currently developing and evaluating an interaction model for navigation systems. Our model facilitates dynamic adaptation to driver intentions and addresses the issues uncovered in this work by improving the credibility of interaction processes.

\section{ACKNOWLEDGMENTS}

The authors would like to thank Björn Wiedersheim for his support during the test drive, the manufacturers and retailers that kindly provided the tested devices, and the anonymous reviewers for their valuable comments. This work was supported by Transregional Collaborative Research Centre SFB/TRR 62 ("Companion-Technology for Cognitive Technical Systems") funded by the German Research Foundation (DFG).

\section{REFERENCES}

[1] R. Dechter and J. Pearl. Generalized best-first search strategies and the optimality of $\mathrm{A}^{*}$. Journal of the ACM, 32(3):505-536, 1985.

[2] E. W. Dijkstra. A note on two problems in connexion with graphs. Numerische Mathematik, 1:269-271, 1959.

[3] B. J. Fogg and H. Tseng. The elements of computer credibility. In CHI '99: Proceedings of the SIGCHI conference on Human factors in computing systems, pages 80-87, New York, NY, USA, 1999. ACM.

[4] R. J. Hanowski, S. C. Kantowitz, and B. H. Kantowitz. Driver acceptance of unreliable route guidance information. In Proceedings of the Human Factors and Ergonomics Society 38th Annual Meeting, volume 2 of System Development, pages 1062-1066, 1994.

[5] A. Malm. Personal navigation devices (3rd edt). Market report, Berg Insight, November 2009. http://www.berginsight.com/ShowReport.aspx?id=92.

[6] The Local. Driver obeys car navigation literally, promptly crashes. http://www.thelocal.de/society/20100423-26745.html, 23 April 2010.

[7] The Times. Sat-nav dunks dozy drivers in deep water. http://www.timesonline.co.uk/tol/news/article707216.ece, 20 April 2006. 